American Journal of Animal and Veterinary Sciences 2 (1): 29-31, 2007

ISSN $1557-4555$

(C) 2007 Science Publications

\title{
Renal and Epididymal Infarctions Associated With Chronic Obstructive Urolithiasis in a Suffolk Ram
}

\author{
${ }^{1}$ Bani Ismail, Z., ${ }^{2}$ Al-Rukibat, R. and ${ }^{3}$ Al-Zghoul, M.B \\ ${ }^{1}$ Department of Veterinary Clinical Sciences, ${ }^{2}$ Department of Pathology and animal Health, ${ }^{3}$ Department \\ of Basic Veterinary Medical Sciences, Faculty of Veterinary Medicine, Jordan University of Science and \\ Technology, Irbid 22110, Jordan
}

\begin{abstract}
A 2-year-old, Suffolk ram with ruptured urethra due to complete obstruction by calculi was treated by tube cystostomy. The surgical procedure was successful in bypassing the obstruction; however the patency of the urethra was never restored. Despite surgical and medical treatment, the condition of the ram was deteriorated and the ram was euthanized. Necropsy identified multifocal renal and epididymal infarcts. Histologically, there were multiple thrombotic small and medium sized arteries through out both kidneys and epididymis. Renal and epididymal infarctions due to thrombotic lesions should be suspected in small ruminants suffering from chronic obstruction of the urethra.
\end{abstract}

Key words: Urolithiasis, infarcts, renal, epididymis, ruminants

\section{INTRODUCTION}

A 2-year-old, Suffolk ram with a 2 week history of dysuria and hematuria was presented to the University Animal Hospital. An obstructive urolithiasis was diagnosed and obstruction was relieved by surgical removal of the urethral process. The ram was discharged from the hospital following the surgical procedure, but was readmitted to the clinic one week after the initial presentation. The ram had been urinating normally after discharge from the hospital started showing signs of dysuria and its systemic condition deteriorated.

On clinical examination, the ram was depressed, and anorexic. Temperature, heart rate, and respiration rate were normal. Ventral abdominal and scrotal edema was evident on palpation. Fluid was oozing from the skin in these areas. Ruptured urethra and urine infiltration of the subcutaneous tissue were confirmed by ultrasonography and urine dip stick test on the oozing fluid that was found to be compatible with urine. Serum chemistry profile revealed severe uremia and azotemia (elevated BUN and creatinine) commonly found in sheep with urinary tract rupture.

The ram was then administered intravenous isotonic fluid in preparation for surgery. Surgical options discussed with the owner included performing tube cystostomy, urethrotomy, or a salvage procedure (perineal urethrostomy). The owner wished to preserve until 24 hours before its second admission, when it

the breeding ability of the ram and elected tube cystostomy despite a poor prognosis for return to breeding because of the extensive tissue damage and possible stricture of the urethra at the site of rupture. Tube cystostomy was performed as described previously ${ }^{[1]}$. Despite surgical and medical treatment, the ram continued to deteriorate and was euthanized 45 days after surgery.

At necropsy the carcass was in fair body condition. Both kidneys were enlarged up to twice the normal size. The capsule of the right kidney was tightly adhered to the renal cortex and both capsules were thickened. Both kidneys contained multifocal, raised, hemorrhagic infarcts extending wedge-shaped into the medulla (Fig. 1). The pelvis of both kidneys was distended with large amounts of purulent fluid (hydronephrosis and pyelonephritis). The distal $8-\mathrm{cm}$ of the penis was necrotic (Fig. 2). The parietal and visceral preputium and the wall of the distal portion of the urethra were severely thickened with fibrosis. The lumen of the distal urethra was narrowed with a stricture approximately $30-\mathrm{cm}$ caudal to the tip of the penis. Following the stricture was a large, approximately $6 \mathrm{~cm}$ in diameter, distended cavity formed by the necrotic urethra filled with necrotic partially mineralized debris, few small stones and fibrinopurulent fluid. The proximal urethra was completely obliterated by fibrous connective tissue caudal to this distension. The urinary bladder was distended with approximately $700 \mathrm{ml}$ of urine. Multifocal mucosal hemorrhages were randomly

Corresponding Author: Bani Ismail, Z. Department of Veterinary Clinical Sciences, Faculty of Veterinary Medicine, Jordan University of Science and Technology, Irbid 22110, Jordan, Phone: (962)2 720100; Fax (962)2 7201081 
distributed in the urinary bladder and urethra. The head of the epididymis of the right testes contained a sharply demarcated infarct (Fig. 3).

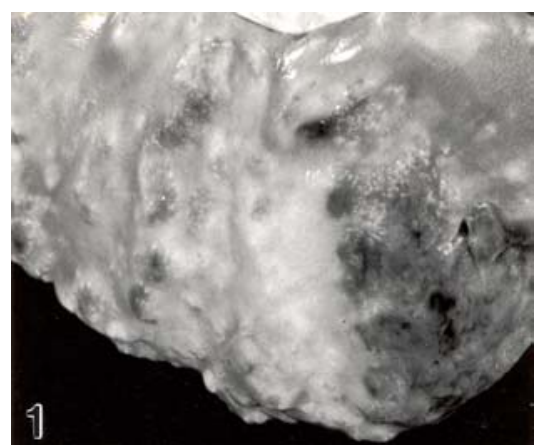

Fig. 1: Renal cortex with multifocal sharply demarcated raised infarcts randomly distributed throughout the parenchyma.

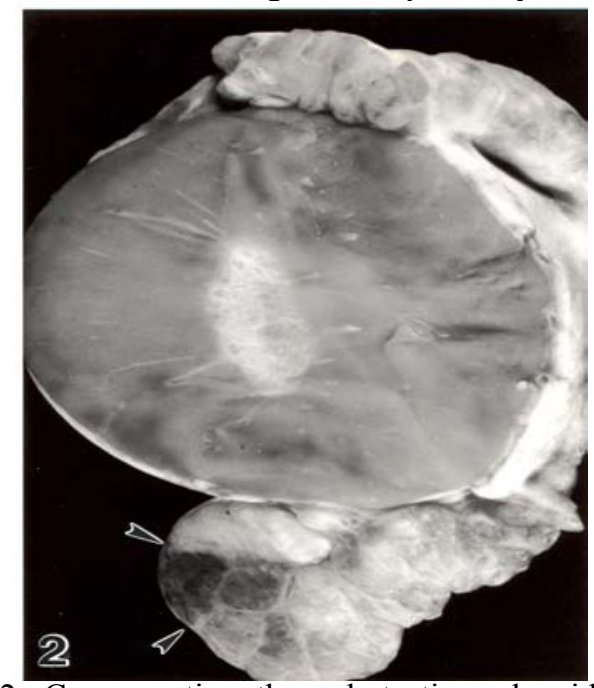

Fig. 2: Cross section through testis and epididymis with sharply demarcated wedge shaped hemorrhagic infarct in the epididymis (arrows).

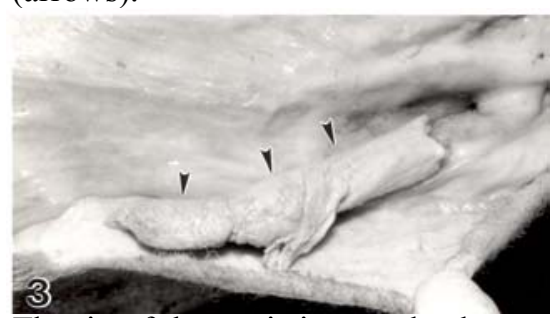

Fig. 3: The tip of the penis is completely necrotic and the opened parietal preputium is severely thickened with fibrosis

Histologicaly, both kidneys had a severe subacute to chronic suppurative pyelonephritis characterized by distention of the renal pelvis with purulent material and suppurative inflammation and necrosis of the calyces associated with areas of tubulointerstitial inflammation and necrosis. Throughout the cortex of both kidneys was multifocal thrombotic occlusion of medium sized arteries associated with a wedge of coagulative necrosis of renal parenchyma (Fig. 4). In the outer margins of the infarcted areas only a few proximal tubules showed epithelial necrosis and a sharp line of demarcation between infarcted and normal tissue was missing due to marginal hyperemia. More centrally in the infarcted area every proximal tubule was necrotic. Similar, but more demarcated infarcted areas were found in the epididymis and small foci of coagulative necrosis were randomly distributed throughout the testis. Multiple small arteries in the epididymis had a severe acute necrotizing vasculitis and there was early thrombus formation in affected vessels occluding the lumen (Fig. 5). The histologic lesions in the lower urinary tract confirmed the above described gross findings.

\section{DISCUSSION}

Urolithiasis is a well-recognized disorder affecting sheep, goats and other animals ${ }^{[2]}$. The disease has a high incidence in feedlot lambs. It has been estimated that approximately $4 \%$ of all feedlot lamb deaths are attributed to obstructive urolithiasis ${ }^{[3]}$. The pathophysiology is multifactorial. Environmental, nutritional, and hormonal factors are believed to interact to produce uroliths ${ }^{[3]}$. Urolith formation is dependent on nidus formation, supersaturation of urinary calculogenic crystalloid and subsequent salt precipitation and concretion ${ }^{[2]}$. Urethral obstruction is more common in males castrated at an early age with a hypoplastic penis and a narrower urethra as the result of lack of testosterone, which effects the development of these structures ${ }^{[2,4]}$.

Medical treatment has been described with marginal success in relieving the obstruction during early stages of the disease ${ }^{[5]}$. However, once urethral obstruction is complete, surgical intervention becomes warranted ${ }^{[6,7]}$. Failure to relieve urethral obstruction can lead to distension and rupture of urethra or bladder. Rupture of urethra and bladder are the most common complications of chronic obstructive urolithiasis. Hydronephrosis as well as pylonephritis leading to renal failure has been reported in a steer with chronic partially obstructive urolithiasis ${ }^{[8]}$.

While hydroureter, hydronephrosis and pyelonephritis are frequently reported sequelae to chronic urethral obstruction ${ }^{[8]}$, renal infarcts associated with chronic urethral obstruction have not been 
reported in animals. Renal infarcts are areas of coagulative necrosis that result from ischemia due to vascular occlusion due to thrombosis or septic emboli ${ }^{[9]}$. In this case, septic emboli are the likely cause of the renal and epididymal infarcts. Ascending urinary tract infection may have caused pyelonephritis with septic emboli lodging in an arcuate artery resulting in coagulative necrosis of segments of the cortex and medulla as well as the epididymis. Another possible explanation for the infarcts in the kidney and epididymis is vasculitis caused by uremia for a prolonged time. After vascular wall injury and activation of the clotting systems, a thrombus may have been dislodged and circulated to occlude an end artery such as the arcuate artery of the kidney or arteries supplying the epididymis resulting in the formation of large infarcts in these two organs. Arteriolar degeneration and necrosis resulting in thrombosis and infarction is well recognized in uremic animals ${ }^{[10]}$. Uremia has been described to cause endothelial and subendothelial damage with formation of large thrombi in these sites ${ }^{[10]}$.

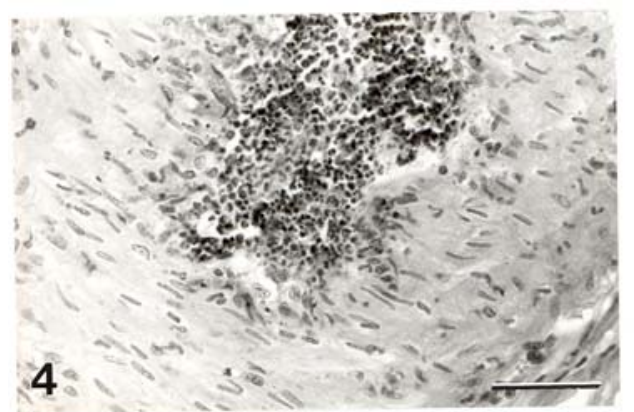

Fig 4: Medium sized muscular artery in the kidney obstructed with cellular debris consisting mainly of necrotic neutrophils. Bar $=100 \mu \mathrm{m}$.

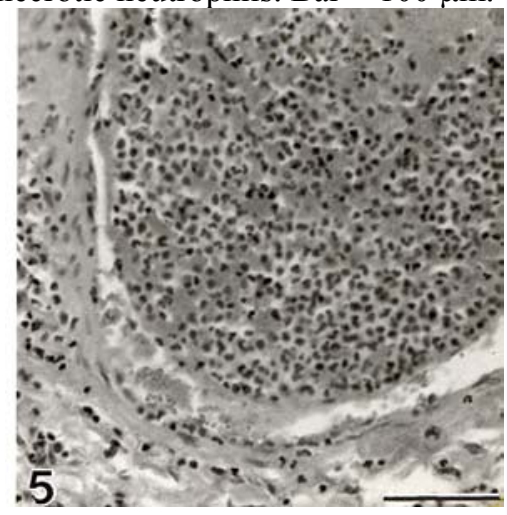

Fig. 5: Small artery in the epididymis with severe vasculitis and early thrombus formation obstructing the lumen. Bar $=100 \mu \mathrm{m}$.

\section{ACKNOWLEDGEMENTS}

The authors would like to thank veterinary students, technicians, clinicians, and staff of the large animal hospital and animal disease diagnostic laboratory of Purdue University for their help in this case management.

\section{REFERENCES}

1. Rakestraw, P.C., Fubini, S.L., Gilbert, R.O., and J.O. Ward, 1995. Tube cystostomy for treatment of obstructive urolithiasis in small ruminants. Am J Vet Surg. 24, 498-505.

2. Blood, D.C. and O.M. Radostitis, 1989. Diseases of the urinary system. In Veterinary Medicine, $7^{\text {th }}$ ed, London, Balliere Tindal., pp: 402-409.

3. Kimberling, C.V. and K.S. Arlond, 1983. Diseases of the urinary tract. Vet Clin North Am Food Anim Pract. 5, 637-655.

4. Bani Ismail, Z. A., Al-Zghoul, M.B., Al- Majali, A.M., and N.M. Khraim, 2007. Effects of castration on penile and urethral development in Awassi lambs. Bulg Vet J. 10, 13-18.

5. Crookshank, H.R., 1970. Effect of ammonium salts on the production of ovine urinary calculi. J Anim Scie. 30, 1002-1004.

6. Haven, M.L., Bowman, K.F., Englebert, T.A., and A.T. Blikslager. 1993. Surgical management of urolithiasis in small ruminants. Cornell Vet. 83, 47-55.

7. House, J.K., Smith, B.P., and L.W. George, 1996. Obstructive urolithiasis in ruminants: Medical treatment and urethral surgery. The Compen Contin Educ Pract Vet. 18, 317-328.

8. Aldridge, B.M., and F.B. Garry, 1992. Chronic partial obstructive urolithiasis causing hydronephrosis and chronic renal failure in a steer. Cornell Vet. 82, 311-317.

9. Confer, A.W., and R.J. Panciera, 1995. The urinary system. In Thompson's Special Veterinary Pathology. Mosby, pp: 209.

10. Maxie M.G., 1985. The urinary system. In Pathology of Domestic Animals. $3^{\text {rd }}$ ed. Orlando: Academic Press, pp: 343. 